\title{
CHONDRODYSTROPHY WITH ECTODERMAL DEFECTS
}

\author{
BY \\ E. J. GALlAGHER, M. E. MACGREGOR and M. ISRAELSKI \\ From the South Warwickshire Hospital Group
}

(RECEIVED FOR PUbLiCATION AUgust 21, 1952)

The following case report is that of a child in whom chondrodystrophy is combined with defects in ectodermal development and who is therefore reminiscent of cases described in 1940 by Ellis and van Creveld. There are, however, interesting differences which cast doubt upon the identity of the syndromes.

\section{Case Report}

M.H., the elder of two girls, first came under observation by one of us (E.J.G.) in 1943 because of multiple orthopaedic deformities. Since then she has received both orthopaedic and plastic surgical treatment which have improved her in some measure both in function and appearance. The following description records her condition in 1950 when aged 12.

Delivery was normal at term. Her mother suffered during pregnancy from hyperemesis, and there is said to have been oligamnios. Birth weight was $7 \frac{1}{2} \mathrm{lb}$. The child progressed well, and, apart from an unexplained short illness at 9 months, her health was good throughout childhood. She talked and walked at the average times, and teeth started to erupt at 8 months. Orthopaedic treatment was begun at the age of 4 , and treatment was carried out at a plastic unit when aged 9.

M.H. is the child of young unrelated parents with no relevant history on either side. The younger child (a girl aged 4 in 1950) is normal.

The patient is an intelligent and cheerful child of average height and nutrition. There is an exaggeration of the normal spinal curvature when she stands, and there is gross knockknee (Fig. 2). Because of stiff joints and deformity of the feet her gait is slow and awkward.

Measurements are as follows: Height, $4 \mathrm{ft}$. 5 in.: weight, $69 \mathrm{lb}$.; head circumference, 20 in.; symphysis to sternal angle, $13 \frac{3}{4}$ in.; chest circumference, 31-
$32 \frac{1}{2}$ in.; interpupillary distance, 2.6 in.; symphysis to vertex, 243 in.; symphysis to sole, 283 in.; limbs (upper), 17 in., (lower right, 303 in., lower left), 291 in.

The skin is generally sallow. There are many areas of brown pigmentation, especially on the face. In pigmented areas, some of which measure several inches in their longer dimension, the skin is puckered and depressed beneath the surface of the surrounding normal skin. At the right outer canthus one such area is responsible for ectropion of the upper lid. One or two of the larger patches have previously been excised. The skin of the nose is pitted by numerous very small depressed patches of this kind. Areas of abnormal skin are largely confined to the face, hands and feet, but one or two puckered areas are to be found on the thighs also.

The mucous membranes are normal, with the exception of the

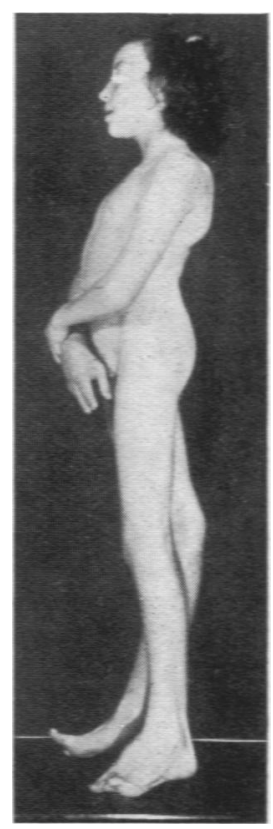

FiG. 2 mucosal surface of the lower lip. Here a gingivolabial sulcus has been fashioned by plastic surgery. Before that the mucous membrane is said to have passed directly from the lower lip to gum without the normal sukcus.

The eyes are widely separated and rather proptotic. The palpebral fissures are oblique, slanting upwards and outwards (Fig. 1).

The nose has large, widely separated nostrils. The ears are normal.

The hair is dark brown and abundant on the scalp, but axillary and pubic hair is scanty.

Only 23 teeth have erupted. They are very irregular in spacing and in lateral alignment. Only one upper incisor is present on each side and three premolars are unerupted. There is a high vault to the hard palate, with the two sides meeting at an obtuse angle in the midline.

The chest is barrel-shaped with, however, good expansion. There is kyphosis of the mid-thoracic region with some scoliosis convex to the left. The spine is flexible. 
As far as the wrist the contour of both upper limbs is normal. There is severe valgus deformity at the right knee. The left leg is $1 \frac{1}{2}$ in. shorter than its fellow, and the thigh and leg circumferences on this side are 1 to 2 in. less. Also there is valgus deformity at the knee and some bowing of the leg, so that the inner border is concave.

There is some limitation of flexion of the right elbow, while extension, pronation and supination of the left elbow are greatly restricted. Left wrist movement is in all directions much reduced. Metacarpo-phalangeal movements are reduced in both hands, while interphalangeal movements are almost completely absent. There are 10 degrees of hyperextension at the right knee with an equivalent loss of full flexion. Otherwise knee movements are full, but the patella is displaced to the outer side of the left knee joint. There is restriction of movement at the left ankle and at the metatarsophalangeal joints on both sides. The interphalangeal joints of toes 2 to 5 on the right side, and of the first and second toes on the left side, are fixed. In the remainder movement is limited.

The hands and feet are greatly deformed (Figs. 3 and 4). Some fingers are flattened in the dorso-ventral plane, and finger length is disordered. Thus the index finger is the shortest in both hands, and the fifth digit the longest. Minor joint deformities, such as acute forward flexion of the terminal phalanx of the right fifth digit, are present besides the limitation in movement already described. Interdigital webbing (up to the distal end of the first phalanx) is present between several fingers, and plastic operations have removed it from others. The left hand has a side-to-side curvature apparently due to puckering and contraction of the skin of the palm. There are also areas of thin, red, shiny, telangiectatic skin over the distal phalanges. The finger nails are dystrophic; that of the fourth right finger is thickened and ridged while those of the thumb and index are flat. The third left finger nail surmounts the end of the finger, with its surface facing distally, and the fourth left nail is also ridged and thickened.

The big toes show considerable valgus deviation and over-ride the neighbouring toes, which are irregular in length, possess interdigital webs, and are closely bunched together. The skin shows numerous callosities, and over the soles is puckered and contracted. Over the lateral aspect of the foot on each side there is a band of telangiectatic skin. The toenails are normal in shape and texture.

There are no abnormal signs in the central nervous or respiratory systems, and the abdomen is normal to palpation. The urine contains no abnormal ingredient.

The heart is not clinically enlarged but a soft diastolic murmur is to be heard at the left border of the sternum at its lower end. The significance of this is uncertain and there was unfortunately no opportunity to amplify this finding. Blood pressure was $135,85 \mathrm{~mm}$. $\mathrm{Hg}$.

X-ray Examinations. These were performed in 1943, 1947 and 1950. when the child was 6,10 and 12 years old respectively.

No convincing enlargement of the heart shadow was seen.

Findings in the bones of hands and feet are very grossly abnormal and pathognomonic of the Ellis-van Creveld syndrome (Figs. 5 and 6). There is a general bone dysplasia resulting in bizarre shapes of most bones. During the years a tendency towards increasing synostoses is evident. The bone age as seen in carpal and tarsal bones and epiphyses is normal.

Right HAND. In 1950 the second to fifth metacarpals were stunted, broad and barrel-shaped. Ankylosis of the inter-phalangeal joints of the second and third fingers was seen with stunted growth of these fingers. A large horseshoe-shaped bone is formed by the synostosis of the second and third metacarpals at their bases, the multangulum minor and the capitate bone.

LeFT HAND. In 1950 it was grossly stunted. The first and second phalanges of the little finger are developed in pairs, all four being fused into a single, oddly shaped bone. A longish bone formation overlying the ulnar edge of the carpel region and fused with the os pisiform probably represents a supernumerary digit. There is also a fusion of the first and second phalanges of the second finger. The inter-phalangeal joints of the second and fourth fingers are almost ankylosed. Very extensive synostoses are to be seen between the carpal bones and the bases of the metacarpals. There is no distinction between the cortex and spongiosa of several of these bones.

FeEt. The second to fifth toes of the right foot are

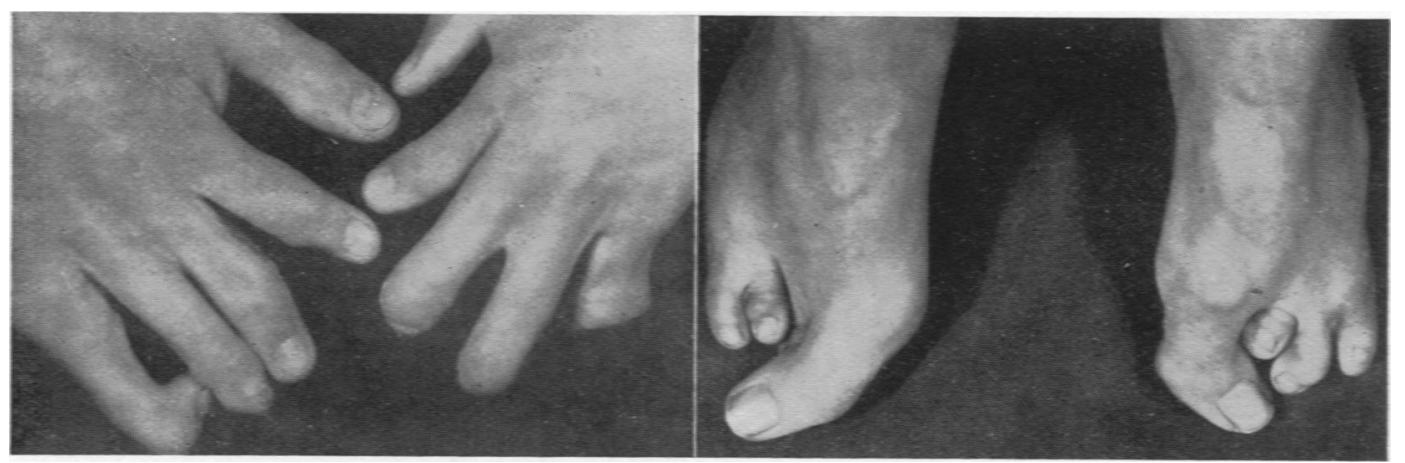

FK. 3.

Fro. 4. 


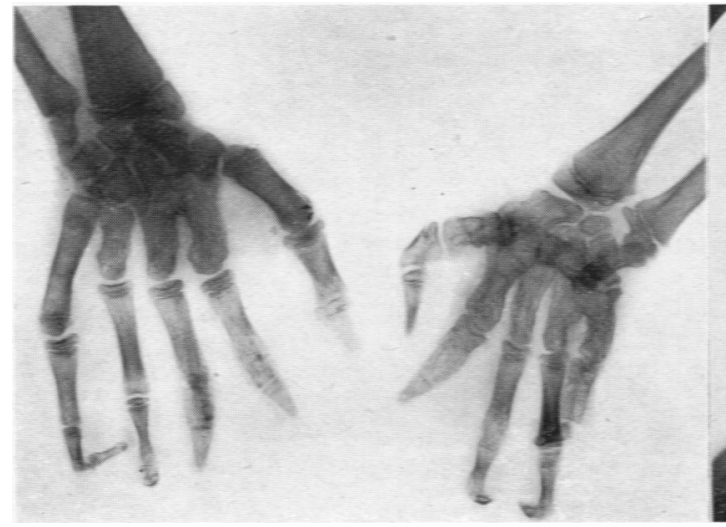

FIG. 5 .

underdeveloped. The metatarsals are very broad, barrelshaped and short. Synostoses between the fifth metatarsal and basal phalanx of the fifth toe, the second cuneiform and the second metatarsal, and talus, calcaneum and cuboid are present.

The left foot shows similar deformities. In addition there is a synostosis involving the third cuneiform and navicular and the second and third metatarsals.

SPINE. The spine shows many features of chondroosteodystrophy (Morquio), which becomes more pronounced as the years advance. In 1943 there was angular kyphosis in the mid-dorsal region, with wedging of two vertebrae which were smooth in outline and regular in texture. All lumbar vertebrae were a little flat but otherwise of good shape. Because of the dorsal kyphosis the antero-posterior diameter of the chest was large. Findings were hardly characteristic at this stage. The wedged vertebrae and dors a 1 kyphosis already suggested some development a l abnormality.

By 1950 all the dorsal and lumbar vertebrae had become markedly flat, and their upper and lower terminal plates very irregular in outline. The upper lumbar and lower dorsal vertebrae show the typical beak-like shape of Morquio's disease. Whereas most discs are a little

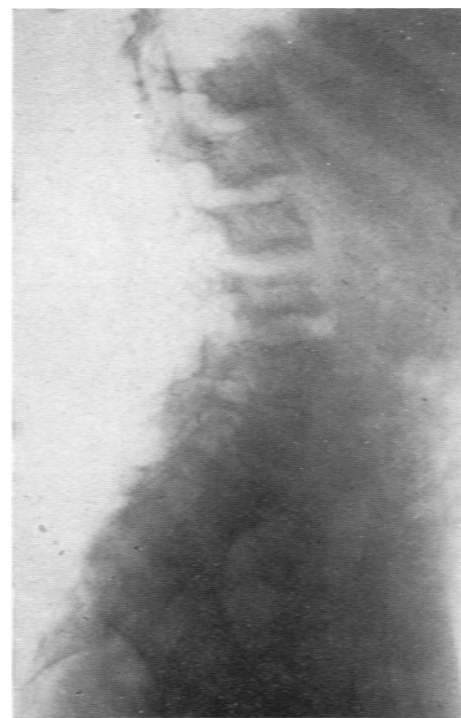

FK. 7. above the normal width, the two lowest dises in the lumbar, and a number in the mid-dorsal, region are narrow or almost absent, indicating that the longitudinal growth of the spine at these levels is about to stop. In the mid-dorsal region there are several almost fully established block vertebrae. This tendency to vertebral fusion clearly distinguishes the findings from those in ordinary Morquio's disease. An unusual lordosis exists in the lower dorsal region (Figs. 7 and 8).

LARGE JOINTS AND LONG BONES. In contrast to the appearances in the spine, the epiphyseal development is generally good and without the features of Morquio's disease. The hips are perfectly normal. So are the epiphyses of the right knee joint. There is a small metaphyseal exostosis at the inner aspect of the right lower femur, the only one found in this case. The left knee shows normal epiphyseal development but a very gross valgus deformity with actual outward subluxation of the lower leg and patella.

The bones of the left leg are atrophic and there are growth lines in femur and tibia. The bones of the lower arms are short and stunted.

Skull. Findings alter little over the years. The skull is large, with no craniostenosis. The increased space between the orbits (hypertelorism) found on clinical 
examination is radiologically very evident. The roofs of the orbits are very much higher than the cribriform plate. There is a steep descent between the floors of the three cranial fossae, a normal sella turcica but rather plump anterior and posterior clinoid processes. The left parietal bone close to the midline shows an area of thinning and outward protrusion (Fig. 9).

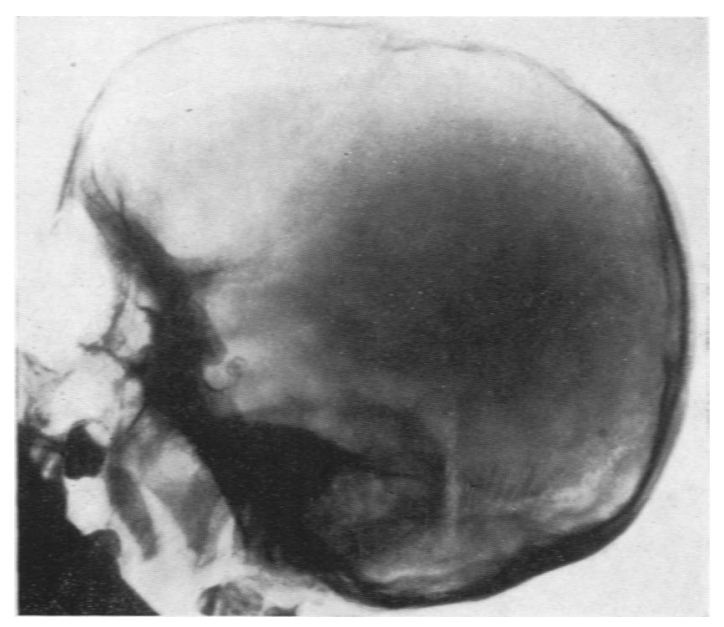

Fr. 9.

\section{Discussion}

Holt and McIntosh in 1933 described the case of a 4-year-old girl, born of Jewish parents who were cousins, and exhibiting polydactyly (six fingers, five toes) and skeletal deformity of the achondroplastic type, with irregular and delayed dentition. There was also internal strabismus and calcification in the submaxillary glands.

In 1940 Ellis and van Creveld reported two similar cases and were able to link these with McIntosh's child as examples of a complex syndrome that they described as chondro-ectodermal dysplasia and which has become known as the Ellis-van Creveld syndrome. Both these cases showed, in addition to polydactyly, ectodermal defects, chondrodystrophy, cardiac enlargement and a murmur. The first, a 20-month-old girl, born prematurely of English cousin parents, had dysplastic nails, sparse hair, deficient and prematurely erupting teeth, an achondroplastic skeleton with exostoses of the hips, six fingers and five toes, a systolic murmur and increased transverse diameter of the heart.

The second, a Dutch boy of $4 \frac{1}{2}$, had no parental consanguinity. His main abnormalities were deficient and late erupting teeth, dysplastic nails, sparse hair, slow growth, six fingers and five toes, achondroplastic limbs with a large head, a large heart with a systolic murmur, a palpable liver and spleen and undescended testicles.

Since then other cases have been collected (Caffey, 1950; Weller, 1951), and while there are minor variations between them, there is an essential sameness of the main abnormalities. Polydactyly and changes of achondroplastic type are present in all. Ectodermal dysplasia is of the hydrotic type, that is, without disturbance of sweat or sebaceous glands. With the exception of Weller's case the intelligence has been normal.

Weller enlarged the concept of this syndrome to include arthrogryposis of phalangeal joints, and published the case of a 2-year-old Turkish girl who demonstrated all five of the primary abnormalities. In this case the parents were not consanguineous and the child was believed to be mentally backward.

The main difference between our case and those previously reported lies in the nature of the bony abnormalities. In the $x$-ray analysis of the Ellisvan Creveld syndrome 'the most constant findings are shortening and deformities of the tibia and fibula, and of the radius and ulna, and shortening of the tubular bones of the hands and feet with polydactyly and massive fusion of the carpal bones. . . . The femur and humerus may be bowed as well as shortened. . . . The spine is normal radiologically ${ }^{\circ}$ (Caffey, 1950). The case here reported, while showing many of the features of the Ellis-van Creveld syndrome, has in addition features of the spinal form of chondro-osteodystrophy (Morquio) associated with a tendency to vertebral fusion.

Unfortunately we lost sight of our patient before a study of the possible heart lesion was complete. Not much significance, therefore, can be attached to the precordial murmur that was audible; with a normal x-ray contour of the heart a congenital heart lesion cannot be substantiated, though it remains a suggestion. From external examination it appears that syndactyly replaces the more usual polydactyly in our case, but a radiograph of the left hand reveals a rudimentary extra digit.

About these differences Ellis (1951) comments:

'I agree that there are several features resembling the cases reported in the Archives, but I think one could hardly say it is the same syndrome. Although one would need many more cases and family histories to speak with any certainty, I should rather imagine that these bizarre syndromes are due to a linkage of genes. If this is so, it would explain why your case shows certain features which differ from ours. The osseous dystrophy, for instance, is more similar to chondro-osteodystrophy than it is to achondroplasia, but at the same time there are undoubtedly intermediary types which show features 
of both. Similarly there are connecting links between syndactyly and polydactyly, though one is primarily a failure of differentiation and the other reduplication or excessive differentiation. I suppose one might reasonably make some more general description which would cover both groups of cases, particularly if the diastolic murmur in your case could be shown to be due to some congenital defect, as I imagine it must be. Thus both groups show abnormalities of differentiation of the digits, ectodermal defects, osseous dystrophy and cardiac abnormality.'

Of the five basic abnormalities in the Ellis-van Creveld syndrome, therefore (polydactyly, ectodermal defects, chondrodystrophy, congenital morbus cordis, arthrogryposis), this child manifests only the ectodermal defects and arthrogryposis in unmistakable form. The characteristic chondrodystrophy and polydactyly are present in rudimentary form, but combined with other obscuring features. The presence or absence of the fifth element, namely, congenital heart disease, remains uncertain.

\section{Summary}

The case of a 12-year-old girl is reported, which exhibits ectodermal defects, spinal chondro-osteodystrophy of the Morquio type and certain other bone and joint deformities.

Resemblances to the Ellis-van Creveld syndrome are discussed.

REFERENCES

Caffey, J. (1950). Pediatric $X$-ray Diagnosis, 2nd ed., p. 675. (Year Book Publishers.) Chicago.

Ellis, R. W. B. (1951). Personal Communication.

and Creveld, S. van (1940). Archives of Disease in Childhood, 15,65 .

Holt, L. E., Jr. and McIntosh, R. (1933). In Holt's Diseases of Infancy and Childhood, 10th ed., p. 362, by the late Holt, L. E. and Howland, J., rev. by Holt, L. E., Jr. and McIntosh R. and How York

Weller, S. D. V. (1951). Proc. roy. Soc. Med., 44, 731. 